\title{
From molecular neurodevelopment to psychiatry: new insights in mechanisms underlying Cannabis-induced psychosis and schizophrenia
}

\author{
Beat Lutz
}

Published online: 2 July 2009

(C) Springer-Verlag 2009

Cannabis with its major psychoactive component, $\Delta^{9}$-tetrahydrocannabinol $\left(\Delta^{9}\right.$-THC), has been in continuous public discussions in the Western world since more than a century. On the one hand, medicinal cannabis has reported beneficial effects in numerous disease states; on the other hand, cannabis is the most widespread illicit drug in the world. The history has seen several cycles of ups and downs, from phases aiming at an unrestricted use (i.e. legal availability like tobacco) to rather prohibited phases (i.e. being a drug classified like opioids).

During the last 20 years, endogenous receptors for $\Delta^{9}$-THC, cannabinoid receptors $\left(\mathrm{CB}_{1}\right.$ receptors in particular for neuronal functions) and endogenous ligands (called endocannabinoids) have been discovered. It has now been recognized that the endocannabinoid system (comprising cannabinoid receptors, endocannabinoids and a synthesizing and degradation machinery for endocannabinoids) represents a novel signalling system in the nervous system, regulating numerous processes, from neural development to synaptic transmission processes. These insights have also enabled a detailed understanding on the pharmacological effects of $\Delta^{9}$-THC in animal models and in humans.

This Special Issue aims at giving clear directions in the public discussion on the potential danger of the recreational use of cannabis, in particular, during pregnancy, puberty and adolescence, in the context of the devastating disorders psychosis and schizophrenia. In addition, it may also define possible undesired effects in medicinal use of cannabis.

\section{B. Lutz $(\square)$}

Institute of Physiological Chemistry and Pathobiochemistry, University Medical Center of the Johannes Gutenberg University Mainz, Duesbergweg 6, 55099 Mainz, Germany

e-mail: blutz@uni-mainz.de
The contribution by Galve-Ropherh et al. discusses recent advances in the understanding of the different and diverse roles of the endocannabinoid system in neural development. Although several investigations reported the presence of cannabinoid receptors during several stages of embryonic development more than a decade ago, the exact functions have been elusive not until recently. At present, the notion is put forward that endocannabinoids are locally produced and are able to regulate neural progenitor proliferation and specification of pyramidal neurons via activation of $\mathrm{CB}_{1}$ receptors. This receptor is also involved in controlling axonal navigation, neuronal migration and positioning of GABAergic interneurons and excitatory neurons. These insights were gained from studies in rodents, where components of the endocannabinoid system were genetically inactivated, or by pharmacological treatments with $\mathrm{CB}_{1}$ receptor antagonists. Besides the exciting insight that endocannabinoids and their receptors are involved in numerous central steps in neural development, these investigations have also shed new light onto the mechanisms underlying the devastating effects of cannabis consumption during critical phases of brain development. As endocannabinoid levels are tightly regulated in time and space to execute their ascribed roles, $\Delta^{9}$-THC consumption leads to a strong disturbance of the endocannabinoid system. $\Delta^{9}$-THC activates all $\mathrm{CB}_{1}$ receptors in the body irrespective of there current intrinsic activation pattern. Secondly, while endocannabinoids undergo a fast degradation, $\Delta^{9}$-THC is rather stable and occupies $\mathrm{CB}_{1}$ receptors for a long time. It is expected that in future studies in animal model systems $\Delta^{9}$-THC-induced disturbances in brain development will obtain distinct molecular signatures, which will help to understand $\Delta^{9}$-THC-induced psychosis and schizophrenia as neurodevelopmental disorders. 
The article by Schneider reviews experiments in rodents where $\Delta^{9}$-THC were applied during pregnancy, puberty or adolescence, inducing permanent and irreversible behavioural changes, such as decreasing the performance in mnemonic processing and increasing drug sensitivity, to some extend changing emotional behaviour and increasing locomotor activity. These developmental phases are particularly vulnerable to $\Delta^{9}$-THC, which presumably interferes strongly with endogenous processes controlled by endocannabinoids and $\mathrm{CB}_{1}$ receptors, such as neuronal migration, axonal pathfinding and synaptogenesis, finally leading to an inappropriate "hardwiring" of the brain. The period of the brain growth spurt (in rats: from birth to postnatal day 21; in humans: third trimester of pregnancy to first 2 years of life) is particularly sensitive to $\Delta^{9}$-THC. Even a single injection of a synthetic $\mathrm{CB}_{1}$ receptor agonist at the peak of the brain growth spurt (i.e. on postnatal day 10 in rats) can lead to long-lasting behavioural changes, including anxiety-related and depressive-like behaviour. Future research will also have to focus on the understanding on the roles of the endocannabinoid system during the phase of the brain growth spurt, as to date, only sparse knowledge has been gained on this period. These studies in rodents are also of importance in the light of the potential therapeutic applications of cannabinoid-based drugs in children (e.g. cancer chemotherapy, ADHD, etc.). Although the reported side-effects were minor, possible long-lasting consequences have not yet been sufficiently investigated.

The review by Jutras-Aswad and colleagues addresses the neurobiological consequences of maternal marijuana use on foetal development in humans. $\mathrm{CB}_{1}$ receptors are highly expressed in mesocoticolimbic areas in the midgestation human foetal brain. These neuronal systems are relevant for mood, cognition, reward and goal-directed behaviours. A majority of studies, in fact, have observed that newborns and infants born to cannabis users have increased tremors, exaggerated startle responses and poor habituation to novel stimuli. By the age of 10 , prenatal marijuana-exposed children were reported to have increased hyperactivity, inattention and impulsive symptoms. Regarding psychiatric disorders, emerging evidence is present that maternal cannabis exposure also predicts early onset and increased frequency of cannabis use. To detail molecular mechanisms underlying this effect, human foetal brain after cannabis exposure showed discrete neuronal disturbances on, e.g. striatopallidal circuits (i.e. decreased expression of proenkephalin and dopamine $\mathrm{D}_{2}$ receptor). Regarding prenatal cannabis exposure and incidence of schizophrenia, further studies with larger cohorts are required, but nevertheless, the current data gives a strong foundation for the public health platform guiding women about the impact of cannabis use during pregnancy. This is not only relevant for recreational cannabis use, but also directed to medical doctors who may prescribe cannabis as an anti-emetic medication to pregnant women.

The contribution by D'Souza discusses the association between cannabis use and psychosis. Clear evidence is provided that acute cannabis treatment can induce transient schizophrenia-like positive, negative and cognitive symptoms in some healthy individuals. The mechanisms underlying these effects may involve dopamine, GABA and glutamate transmission. Regarding the emergence of schizophrenia after frequent cannabis use, only a small proportion of the general population exposed to cannabis develop psychotic illness. D'Souza states that the exposure to cannabis is neither a necessary nor a sufficient cause of schizophrenia. More likely, cannabis exposure is a contributing cause which interacts with other known (e.g. genetic, environmental) and unknown factors, finally leading to schizophrenia. For future studies, it will be necessary to characterize the factors that underlie individual vulnerability to cannabis-induced psychosis. Here, it is hoped that the recent insights on the role of the endocannabinoid system in neural development and in the modulation of neurotransmitter systems may lead to new vistas on such factors.

The contributions to this Special Issue represent an interdisciplinary approach discussing the aspect for cannabis and psychosis from basic research to the clinic. A future challenge in the field will be to define clear molecular identities for cannabis-induced psychosis and schizophrenia. Here, a further understanding on the role of the endocannabinoid system in neural development is needed, with complementary insights from the pathology of schizophrenia at the molecular level.

Acknowledgments The guest editor wishes to thank all the persons who significantly contributed to this issue, namely all the authors, and Prof. Dr. Heinz Häfner for the kind invitation and his continuous support to generate this Special Issue. 